\title{
Elimination of Lymphatic Filariasis: A Neglected Disease of India
}

\author{
Wahied Khawar Balwan ${ }^{1 *}$, Neelam Saba $^{2}$ \\ ${ }^{1}$ Department of Zoology, Govt. Postgraduate College Bhaderwah, Jammu \& Kashmir, India \\ ${ }^{2}$ Department of Zoology, Govt. Degree College Doda, Jammu \& Kashmir, India
}

\begin{abstract}
Article History
Received: 27.01.2021

Accepted: 16.03 .2021

Published: 23.03.2021

Journal homepage:

$\underline{\text { https://www.easpublisher.com }}$

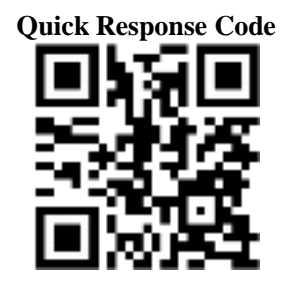

Abstract: In India, human lymphatic filariasis is the most common vector borne disease after malaria. Lymphatic Filariasis caused by nematode worms Wuchereria bancrofti and Brugia malayi is a major health problem in India, which afflict mostly poor people. The disease leads to disfiguring pathological conditions, severe social stigma, psychological problems and huge economic losses on affected individuals and communities. Filariasis is a major public health problem in India and inspite of existence of the National Filaria Control Programme since 1955, currently there may be up to 31 million microfilaraemics, 23 million cases of symptomatic filariasis, and about 473 million individuals potentially at risk of infection. Over the last 10 years advances have led to new diagnostic / treatment tools and control strategies for filariasis. With the advent of new and easy to implement control strategies, Lymphatic filariasis elimination programs have gained much momentum in the past decade but there are still many challenges that stand in the way of achieving the goal of Lymphatic filariasis free India. A special emphasis has been given on the general hygiene and environmental management of mosquito vectors under the Swachh Bharat Mission (Clean India Movement) and also to provide special incentive under the Ayushman Bharat to make the programme effective and successful.
\end{abstract}

Keywords: Lymphatic filariasis, Wuchereria bancrofti, Brugia malayi.

Copyright (C) 2021 The Author(s): This is an open-access article distributed under the terms of the Creative Commons Attribution 4.0 International License (CC BY-NC 4.0) which permits unrestricted use, distribution, and reproduction in any medium for non-commercial use provided the original author and source are credited.

\section{INTRODUCTION}

Filariasis has been a major public health problem in India. The disease was recorded in India as early as $6^{\text {th }}$ century B.C. by the famous Indian physician, Susruta in his book 'Susruta Samhita' [1]. The neglected tropical diseases are a group of 13 major disabling conditions that predominantly affect world's poorest people in Africa, Asia and the Americas. Lymphatic filariasis, caused by filarial nematodes $W$. bancrofti and B. malayi and transmitted by mosquitoes, is one of the neglected tropical diseases which is prevalent in 81 tropical and subtropical countries. It is estimated that approximately 1.3 billion world population is at risk of filarial infection. About 129 million people are infected with Lymphatic filariasis and among them 40 million are seriously incapacitated and disfigured. According to World Health Organisation (WHO), Lymphatic filariasis is the second most cause of long term disability after mental illness. One third of people infected with Lymphatic filariasis live in India, one third live in Africa and most of the remainder lives in the Americas, the Pacific Islands and South-East Asia. The burden of Lymphatic filariasis in humans and its impact on socioeconomic aspects has led to the identification of this disease as one of the priority areas of WHO. In the year 1997, the World Health Assembly at its 50th session passed a resolution (WHA 50.29) to eliminate Lymphatic filariasis globally as a public health problem by the year 2020 [2]. The two main objectives of Global Program to Eliminate Lymphatic filariasis (GPELF) are (1) interruption of transmission of parasite through repeated annual mass drug administration (MDA), using a combination of ivermectin plus albendazole where oncocerciasis is coendemic with Lymphatic filariasis and diethylcarbamazine plus albendazole where Lymphatic filariasis alone is endemic, to all the people living in endemic area and (2) prevention of Lymphatic filariasis related disability through morbidity management program. GPELF is benefitted by generous donation of drugs Albendazole by GlaxoSmithKline and Ivermectin by Merck and Co., Inc. as long as they are required to eliminate Lymphatic filariasis.

\section{Current Status of Lymphatic Filariasis in India}

Lymphatic filariasis is a major health problem in India and is endemic in 250 districts of 20 states/union territories [3]. In India, W. bancrofti transmitted by the ubiquitous vector, Culex uinquefasciatus, has been the most predominant infection contributing to $99.4 \%$ (bancroftian filariasis) of filarial cases. The infection is 
prevalent in both urban and rural areas while rest of the infections are caused by B. malayi (brugian filariasis), which is transmitted by Mansonia mosquitoes. Brugian filariasis is mainly restricted to rural areas of Uttar Pradesh, Bihar, Andhra Pradesh, Odhisa, Tamil Nadu, Kerala and West Bengal. The incidence of Lymphatic filariasis in India is very high, currently over 45.5 million people infected with Lymphatic filariasis lives in India and about 553 million are exposed to the risk of infection, of these about 146 million live in urban areas and about 407 million in rural areas. The state of Bihar has highest endemicity of over $17 \%$ followed by Kerala (15.7\%) and Uttar Pradesh (14.6\%). Andhra Pradesh and Tamil Nadu have about $10 \%$ endemicity. Goa showed the lowest endemicity of less than $1 \%$ followed by Lakshadweep (1.8\%), Madhya Pradesh (above 3\%) and Assam (about 5\%). The seven states namely Andhra Pradesh, Bihar, Kerala, Odisha. Uttar Pradesh, Uttrakhand and West Bengal contribute $86 \%$ of microfilaria carriers and $97 \%$ of disease cases in the country [4].

\section{Symptoms and Socio Economic Burden}

Symptoms of Lymphatic filariasis can be divided into three basic disease stages namely (a) asymptomatic (b) acute and (c) chronic. Asymptomatic microfilaraemia is often regarded as a non-disease because the individuals concerned have no idea that their blood contains large numbers of microfilariae and this situation may persist for decades without any progression to clinical disease. Most of the signs and symptoms of filariasis are caused as a consequence of the adult worms living in the lymph system. Tissue damage caused by the worms restricts the normal flow of lymph fluid resulting in swelling, scarring, and infections. The most common manifestation of acute filariasis is adeno-lymphadenitis (ADL), which is characterized by intense lumphangitis, lymphadenitis and reddening of the overlying skin. The worst symptoms of the chronic disease generally appear in adults, include persistent lymphoedema of arms and legs, hydrocoel, elephantiasis of genital organs, chyluria (milky urine) and tropical pulmonary eosinophilia. In addition, Lymphatic filariasis also causes internal damage to the kidneys [3].

Chronic filarial infections have serious social, psychological and economic effects. The massive swelling of the limbs and disfigurement due to lymphoedema interferes with the day-to-day activities of the sufferers and reduces their productivity resulting in low income and long term poverty. The problem becomes more intense, if the patient is major income earner of the family. In rural areas, where agriculture is the primary source of livelihood, incidence of Lymphatic filariasis affects the agricultural activity of farmers leading to poor harvest, loss of livelihood and food insecurity. It is estimated that the total disability adjusted life years lost in India due to this disease are around 2.06 million, resulting in an annual wage loss of \$ 1 billion [5]. Lymphatic filariasis also exerts a profound social burden on patients as the chronic manifestations of this disease such as lymphoedema of the limbs, breasts and external genitalia cause social stigmatization of the patients and prevent them from playing their role in society.

\section{Life Cycle and Pathogenesis}

Human filarial nematode worms have a complicated life cycle, which requires both a vertebrate host and a blood sucking arthropod vector (Figure-1). Human is the definitive host and arthropod vector is the intermediate host. There are no intervening free living stages. Microfilariae are picked up by arthropod vector during their feeding on the infected person. Inside the intermediate host these microfilariae lose their sheath and migrate rapidly to the thoracic muscles where they develop into first stage larvae and subsequently into third stage larvae and migrate through the haemocoel to the mosquito's proboscis. These third stage larvae require human host for their further development. The third stage larvae enter into the human host during a blood meal by an infected mosquito and penetrate into the blood capillaries. Inside human host third stage larvae molt to fourth stage larvae in approximately 4-6 weeks. Within nine months they molt again to the sexually mature juvenile adult stage. The adult filarial parasites reside in the lymphatics, where they can live upto 15 year. The worms have and estimated active reproductive span of 4-6 years. The female worms measures 80 to $100 \mathrm{~mm}$ in length and 0.24 to $0.30 \mathrm{~mm}$ in diameter, while the shorter males measure about 40 $\mathrm{mm}$ by $0.1 \mathrm{~mm}$. The mature adult male and female parasite mate and produce millions of very small immature larvae known as $\mathrm{mf}$ that find their way into the blood circulation. These mf measures 244 to 296 $\mu \mathrm{m}$ by 7.5 to $10 \mu \mathrm{m}$, which are sheathed and have nocturnal periodicity. These are essentially pre-larval stages, which will not undergo further development until taken into the haemocoel of the intermediate host. 


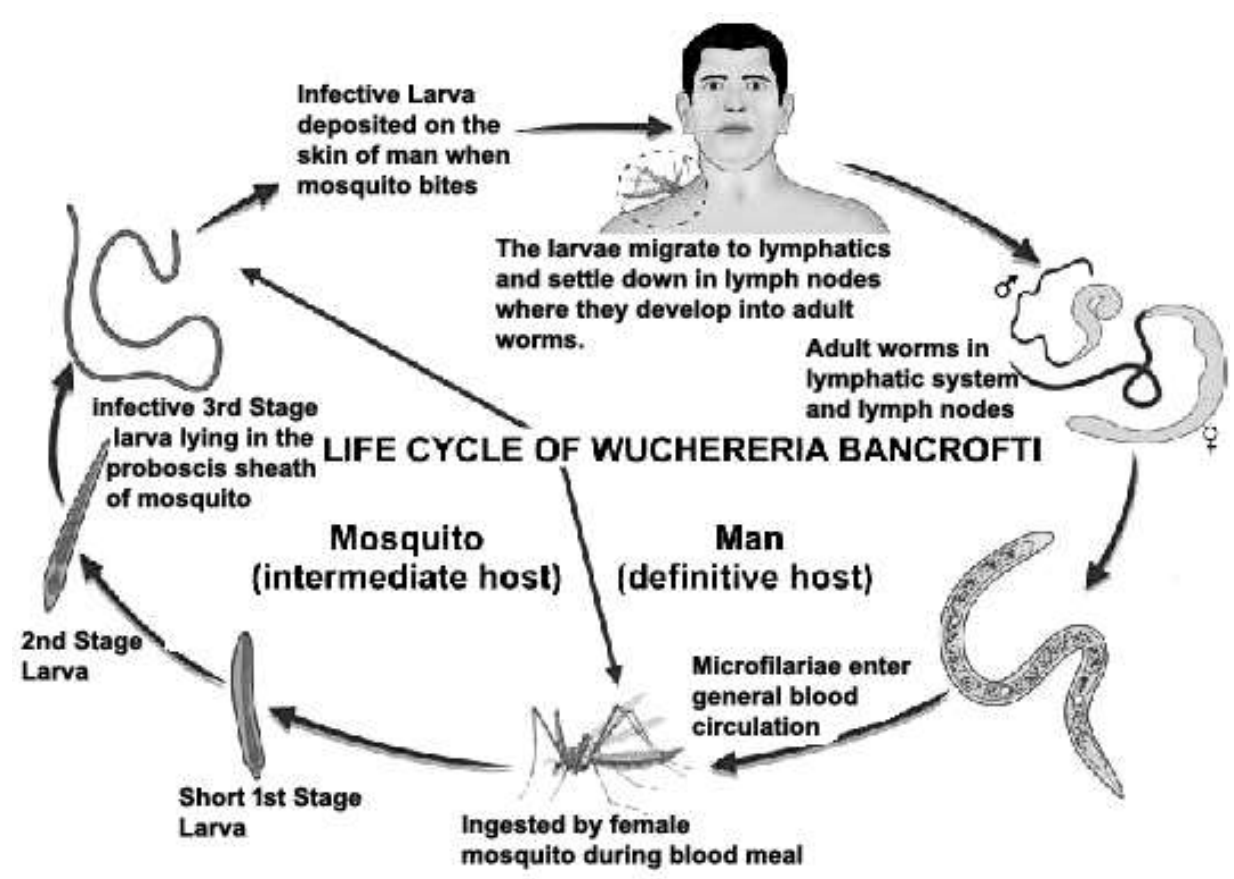

Fig-1: Life Cycle of Filarial Parasite

\section{Present Scenario of Lymphatic Filariasis Elimination in India}

Considering the impact of Lymphatic filariasis on the affected population, National Filaria Control Program (NFCP) was launched in India in 1955 with the objective of delimiting the problem and to undertake control measures in endemic areas. Initially NFCP activities were mainly confined to urban areas, which were implemented through 206 control units and 199 filaria clinics. However, the program was extended to rural areas in 1996 in 13 districts of 7 endemic states through annual mass drug administration (MDA) with single dose of diethylcarbamazine, covering 41 million populations. The program was scaled up to cover a population of 77 million in 2002 with the administration of diethylcarbamazine alone in 19 districts and combination of diethylcarbamazine and albendazole in 11 districts [6]. In 2002, the National Health Policy has set the goal of elimination of Lymphatic filariasis as a public health problem in India by 2015 . For achieving this goal, mass drug administration with annual single dose of diethylcarbamazine $(6 \mathrm{mg} / \mathrm{kg}$ body weight) for five or more years to all the population vulnerable to filariasis, excluding pregnant women, children below 2 years of age and seriously ill persons, was launched in 2004 targeting about 468 million populations from 202 districts. It was also proposed to observe National Filaria Day every year from 2004 in all endemic districts. The coverage of mass drug administration has been reported to be $72.6 \%, 79.84 \%$ and $83.67 \%$ respectively in the year 2004, 2005 and 2006 respectively. From 2007, the program was scaled up to cover the entire population of 590 million in all 250 endemic districts. Administration of ALB (400 mg alongwith diethylcarbamazine (DEC) was included in mass drug administration from 2008 onwards. Besides
MDA another pillar for elimination of Lymphatic filariasis is morbidity management, which includes home based management of lymphoedema cases by simple washing and surgical intervention for hydrocele cases.

Major Problems and Challenges for Disease Control With the advent of new and easy to implement control strategies, NFCP has gained much momentum in the past decade but there are still many challenges that stand in the way of achieving the goal of Lymphatic filariasis free India. It is evident from number of studies that some MDAs work better and more efficiently than the others [7, 8]. The major challenge of MDA is that it requires very high treatment coverage and compliance $(>85 \%)$ sustained for consecutive five years, which is believed to be average reproductive life of adult worms, for achieving transmission interruption of the disease in endemic areas [9]. However many MDAs have struggled to attain this required level of coverage was found below the optimum levels in spite of several rounds of MDAs with social mobilization in Tamil Nadu [10] and Andhra Pradesh states [11]. The study conducted in Bihar district of Karnataka showed that only $62.3 \%$ coverage and $60.4 \%$ compliance could be achieved with MDA, which was much below the expected national standard [12]. The other study in Paschim Midnapur district of West Bengal over a two year period from 2009 to 2010 also indicated low coverage $(84.1 \%$ in 2009 and $78.5 \%$ in 2010) and compliance $(70.5 \%$ in 2009 and $66.9 \%$ in 2010) [13]. Similar findings of low compliance ranging from $32.7 \%$ to $76.2 \%$ were also observed by other studies across India $[14,15]$. Some of the main reasons, which were cited for insufficient coverage and compliance include: lack of knowledge 
about the disease and the program among the endemic population, inconsistent drug distribution, inadequate training of health workers and their reluctance in drug distribution, lack of supervised dosing, fear of side effects of drug, incapability of health personnel to convince people, who feel healthy and have no sign of disease, for drug consumption and absence of recipients of drugs at the time of MDA [16, 17]. In addition, MDAs have also been affected by inadequate supply of drug, insufficient time for MDA implementation and storage of health workers, and repeated postponement of MDA. It has been seen that inadequate compliance is a major impediment to elimination campaign as the non-compliant persons remain microfilaremic and act as a reservoir of infection and plays very important role in resurgence of disease [18-20]. The other challenge to the elimination program is the dearth of anti-filarial drugs, currently DEC alone or combination of albendazole (ALB) and DEC is distributed in MDA programs. These drugs are principally mirofilaricidal and are not able to kill adult worms and provide only partial benefit to the patient. Moreover, there prolong use for number of years to prevent the build-up of $\mathrm{mf}$ from the surviving adult worm has raised the concern about emergence of resistance [20, 2].

\section{Future Perspective}

The success of elimination program depends on sustained and sufficient compliance with MDA rather than MDA coverage. Most of the studies in India indicated that MDA was restricted to tablet distribution only and the major issues of implementation in compliance, in health education, social mobilization, morbidity management and the logistics were not been given due attention. Some imperative steps are therefore urgently needed to improve strategy of MDA implementation through cooperation and coordination of government officials, local health workers, nongovernmental organizations and community volunteers. The reluctance of health personnel or drug distributors (DDs) to strictly adhere to the national guidelines for program implementation, which has been cited as the main cause of low compliance and coverage in most of the studies, warrants some strategic changes in drug delivery mechanism so that drug can be best delivered to the mouth of endemic population. Further, current MDA programs should be supplemented with educational intervention and motivational activities at national level as well as at community level so that maximum number of persons can be informed about cause, transmission and elimination strategy for Lymphatic filariasis. As DDs interact directly with the population living in endemic areas therefore emphasis should be given on recruitment of well trained, motivated and enthusiastic DDs, who can convince the people for drug consumption. For training of health workers educational camps must be organized at national level with the involvement of educational institutes. More importantly, drug consumption should be monitored directly by the health workers as insufficient compliance leads to recurrence of microfilaraemia in the affected individuals and also place the community under risk of filarial infections, which in turn necessitates more rounds of MDA and additional fund for the implementation of program. Vector control has played important role in filariasis control in some programs but it has been given very low priority in India. Therefore, in situation where transmission interruption is not possible through MDA alone the role of integrated vector management as a potential supplementary strategy needs to be explored. Further, adequate monitoring and surveillance is also required to determine the new foci in non-endemic areas as these can serve as sites for fresh infection. Though, many research studies discussed here have failed to implement MDAs successfully but they provide some important clues for the development of more effective drug delivery strategies $[2,21]$.

Furthermore, drugs for Lymphatic filariasis are taken for years therefore in consideration of threat of emergence of drug resistance also to overcome the wellknown deficiencies of the existing drugs, policies should be made to create more funds for research and development of new, safe and more effective antifilarial drugs and vaccines. Despite huge detrimental effects on affected individuals and communities, Lymphatic filariasis is given very low priority and in fact the disease has escaped the attention of planners and policy makers because of low mortality rate and its association with poverty. The NTDs including Lymphatic filariasis occurs mostly among people living on less than $\$ 2$ per day. The big pharmaceutical companies do not take initiative to embark on research and development activities for Lymphatic filariasis because of low profit as the people who need new vaccine and treatments the most can never afford to pay for it. The analysis of the outcomes of pharmaceutical research and development over the past 25 years revealed that out of 13,000 chemical entities marketed between 1975 and 1999, only thirteen were for (Neglected Tropical Diseases)NTDs, of these only three combinations of Ivermectin (IVM) plus ALB; IVM plus doxycycline and ALB plus DEC were registered for LF. With an impressive growth of healthcare industry in the past decade, India has emerged as an innovative developing country which has the capacity of producing its own drugs, vaccines and diagnostics. Therefore, India urgently needs new strategy to stimulate drug research and development program for NTDs through the establishment of public-private partnership of leading government institutions and biopharmaceutical companies [22, 21, 23].

The target set by GPELF in 2000 to eliminate Lymphatic filariasis as a public health problem globally by 2020 will not be achieved by then. Despite setbacks due to COVID-19, WHO will accelerate work to achieve this target by 2030. New global estimates suggest a $74 \%$ reduction in the number of infected 
people since the start of GPELF. The new, ambitious targets for 2030 are that $80 \%$ of endemic countries have met the criteria for validation of elimination as a public health problem, with the remaining $20 \%$ under posttreatment surveillance, meaning that MDA will no longer be required.

GPELF aims to reduce the prevalence of infection below target thresholds and to alleviate the suffering of people affected by lymphoedema and hydrocele, the chronic manifestations of the disease. The recommended essential package of care for managing lymphoedema and hydrocele should be available in $100 \%$ of districts where people are living with these manifestations. This goal is aligned with the aims of universal health coverage to leave no one behind by 2030. Reporting of Lymphatic filariasis morbidity improved again in 2019, showing that countries are addressing this aim and planning services for people affected by the disease.

In 2019, 538.1 million people were treated for lymphatic filariasis (LF) in 38 countries that implemented mass drug administration (MDA) of populations at risk of the disease, as recommended by the World Health Organization (WHO). Seventeen countries achieved the criteria for elimination of LF as a public health problem; Kiribati, Malawi and Yemen were the latest to be acknowledged by WHO. The Global Programme to Eliminate Lymphatic Filariasis (GPELF) has delivered over 8.2 billion cumulative treatments to more than 923 million people since 2000 . The treatments target the parasites in the blood of infected people and prevent the risk of transmission in the community. Infections have been brought to such low levels in some areas that 649.1 million people no longer require MDA for this debilitating parasitic disease.

\section{Risks to Success}

In 2017, WHO published a new guideline on alternative MDA regimens to eliminate LF and recommended a combined regimen of ivermectin, diethylcarbamazine citrate and albendazole (IDA) to accelerate the impact of MDA on transmission of the parasites. In 2019, IDA was used to treat 45.2 million people in 11 countries. In India, IDA was extended from four to 16 districts to treat more than 41 million people. Currently, only six of 17 countries validated as having eliminated Lymphatic filariasis as a public health problem report surveillance activities. Without robust post-validation activities, transmission can remain undetected and the number of infections can resurge to previous levels. Countries are willing to conduct surveillance and remain vigilant, but programmes require clear guidance and resources. Additional research and better diagnostics are necessary to design more detailed, standardized methods for surveillance.

\section{CONCLUSION}

In spite of high morbidity, Lymphatic filariasis remains neglected and understudied as compared to other infectious diseases such as tuberculosis, HIV/AIDS and malaria. Because of the extent of disease problem and its negative impact on affected individuals, some concrete steps should be initiated soon to remedy this gruesome disease. These include to:

1. Motivate the community to participate in the MDA program by raising general awareness about the cause and transmittance of the disease

2. Educate the communities about cleanliness as inadequate sanitation creates numerous breeding sites for the mosquitoes that transmit the disease

3. Earmark the funds for research and development of new, safe and affordable treatment regimens, most important macrofilaricidal drugs

4. Plan and implement MDA program systematically and efficiently to the entire country within a timeframe without any inconsistency so that required level of coverage and compliance could be achieved

5. Generate resources importantly consistence funding for proper functioning of MDAs.

In addition, the elimination programs must focus on morbidity management and disability prevention, so that the diseased persons could be able to live self-dependent and respectful life. Furthermore, tremendous efforts are also required by policy makers, program manager, governmental and non-governmental organizations, health workers, community volunteer and individuals residing in endemic areas for the accomplishment of MDAs leading to the success of NECP. Though the filarial elimination program in India have geared up a lot during the past decade to make its presence felt worldwide, the Indian government needs to act swiftly in a time-bound manner if it has to achieve the target of eliminating Lymphatic filariasis in the country. India is on a very strong ground to achieve lymphatic elimination. Several efforts are now in place. The total disability adjusted life years (DALYs) lost due to Lymphatic filariasis is around 2.06 million, resulting in an annual wage loss of US \$2.06 million, resulting in an annual wage loss of US $\$ 811$ million. A special emphasis has been given on the general hygiene and environmental management of mosquito vectors under the Swachh Bharat Mission (Clean India Movement) and also to provide special incentive under the Ayushman Bharat to make the programme effective and successful.

Conflict of Interest: The authors declare no conflict of interest. 


\section{REFERENCES}

1. Bhaskar, C., \& Harinath, M. V. R. (2000). Filariasis in India. Journal International Medical Science Acadamy, 13:8-12.

2. Katiyar, D., \& Singh, L. K. (2011). Filariasis: Current status, treatment and recent advances in drug development. Current Medicinal Chemistry, 18: 2174-2185.

3. Shenoy, R. K. (2008). Clinical and Pathological aspects of filarial lymphedema and its management. Korean Journal of Parasitology, 46: 119-125.

4. Katiyar, D. (2016). Lymphatic Filariasis: A neglected disease of India. Everyman Science, L (5):295-300.

5. Ramaiah, K. D., Das, P. K., Micheal, E., \& Guyatt, H. (2000). The economic burden of lymphatic filariasis in India. Parasitol Today, 16(6): 251253.

6. National Vector Borne Diseases Control Program; Problems and elimination of lymphatic filariasis in India. Government of India, Ministry of Health and Family Welfare: New Delhi, 2005.

7. Mehta, S., Shah, V., Verma, A., Patel, N., \& Bansal, R. (2012). Comparison of coverage and compliance of mass drug administration 2012 in surat, India. National J Community Med, 3(3):448472.

8. Srivastava, P. K., \& Dhillon, G. P. (2008). Elimination of lymphatic filariasis in India. $J$ Indian Med Assoc, 106:673-677.

9. Government of India. Operational guidelines on elimination of lymphatic filariasis. Delhi: Directorate of National Vector Borne Disease Control Programme, 2005. http://nvbdcp.gov.in/doc/guidelines-filariasiselimination-india .pdf.

10. Nandha, B., Krishnamoorthy, K., \& Jambulingam, P. (2013). Towards elimination of lymphatic filariasis: Social mobilization issues and challenges in mass drug administration with antifilarial drugs in Tamil Nadu, South India. Health Education Research, 28(4): 591-598.

11. Mukhopadhyay, A. K., Patnaik, S. K., \& SatyaBabu, P. (2008). Knowledge on lymphatic filariasis and mass drug administration (MDA) programme in filaria endemic districts of Andhra Pradesh. J Vector Borne Dis, 45: 73-75.

12. Koradhanyamath, D. M., Kulkarni, P., \& Holla, R. (2012). Coverage and compliance MDA programme for lymphatic filariasis. Asian Pacific Journal of Tropical Disease, 2(4): 290-292.

13. Sinha, N., Malik, S., Panja, T. K., \& Haldar, A.
(2012). Coverage and compliance of mass drug administration in lymphatic filariasis: a comparative analysis in a district of West Bengal, India. Global Journal of Medicine and Public Health, 1(1):1-10.

14. Singh, S., Bora, D., Dhariwal, A. C., Singh, R., \& Lal, S. (2006). Lymphatic filariasis in rural areas of Patna district, Bihar. A challenge ahead. $J$ Common Dis, 38(2): 160-163.

15. Gupta, A., Prasad, P., \& Singh, S. P. (2003). A study of coverage and compliance of mass drug administration for elimination of lymphatic filariasis in Rewa district of Madhya Pradesh. National Journal of Community Medicine, 5(1): 38-41.

16. Babu, B. V., \& Satyanarayana, K. (2003). Factors responsible for coverage and compliance in mass drug administration during the programme to eliminate lymphatic filariasis in the East Godavari District, South India. Trop Doct, 33(2): 79-82.

17. Ranganath, B. G. (2010). Coverage survey for assessing mass drug administration against lymphatic filariasis in Gulbarga district, Karnataka, India. Journal of Vector Borne Diseases, 47(1): 61-64.

18. Upadhyayula, S. M., Mutheneni, S. R., Kadiri, M. R., Kumaraswamy, S., \& Nagalla, B. (2012). A cohort study of lymphatic filariasis on socio economic conditions in Andhra Pradesh, India. PloS one, 7(3), e33779.

19. Ramaiah, K. D., \& Vanamail, P. (2013). Surveillance of lymphatic filariasis after stopping ten years of mass drug administration in rural communities in South India. Trans $R$ Soc Trop Med Hyg, 107(5): 293-300.

20. Hotez, P. J., Molyneux, D. H., Fenwick, A., Kumaresan, J., Sachs, S. E., Sachs, J. D., \& Savioli, L. (2007). Control of Neglected Tropical Diseases. New Engl J Med, 257:1019-1027.

21. Chand, G., Barde, P. V., \& Singh, N. (2013). Emergence of new foci filariasis in Madhya Pradesh, India. Transactions of the Royal Society Tropical Medicine and Hygiene, 107(7): 462-464.

22. Sabesan, S., Vanamail, P., Raju, K. H. K., \& Jambulingam, P (2010). Lymphatic Filariasis in India: Epidemiology and control measures. $J$ Postgrad Med, 56(3): 232-238.

23. Trouiller, P., Olliaro, P., Torreele, E., Orbinski, J., Laing, L., \& Ford, N. (2002). Drug development for neglected diseases: a deficient market and a public health policy failure. Lancet, 359(9324): 2188-2194.

Cite This Article: Wahied Khawar Balwan \& Neelam Saba (2021). Elimination of Lymphatic Filariasis: A Neglected Disease of India. EAS J Parasitol Infect Dis, 3(2), 31-36. 